\title{
LIDERAZGO EFICAZ PARA LA MEJORA ESCOLAR. UN ESTUDIO DE CASO DEL PROGRAMA ESCUELAS DE CALIDAD EN EL CENTRO DE MÉXICO
}

\author{
EFFECTIVE LEADERSHIP FOR \\ SCHOOL IMPROVEMENT. A CASE STUDY \\ OF THE QUALITY SCHOOLS \\ PROGRAM IN CENTRAL MEXICO
}

José Matías Romo Martínez

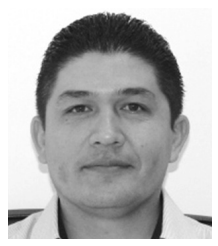

Doctor en Ciencias, con especialidad en Investigación Educativa, Departamento de Investigaciones Educativas, Centro de Investigación y de Estudios Avanzados del Instituto Politécnico Nacional DIE-CINVESTAV. Maestro en Educación y Licenciado en Psicología, Universidad Autónoma de Aguascalientes. Actualmente es profesor de Tiempo Completo en la Universidad Pedagógica Nacional, Aguascalientes y ha colaborado en el Instituto Nacional para la Evaluación de la Educación. Realizó una estancia de investigación en el CREA, Universidad de Barcelona. Miembro del Sistema Nacional de Investigadores (SNI), del Consejo Mexicano de Investigación Educativa (COMIE) y de la Red de Educación y Valores (REDUVAL).

Correo electrónico: [matias.romo@yahoo.com.mx].

\section{RESUMEN}

En México, la desigualdad en la oferta de oportunidades educativas aún es un gran reto. Entre las políticas y programas implementados destaca el Programa Escuelas de Calidad (PEC), que inició hace diez años como una propuesta de reforma institucional y cambios en la 
gestión educativa. Se realizó un estudio de caso con una escuela exitosa inscrita en este programa, la cual se sitúa en una localidad rural del estado de Aguascalientes, México. El acercamiento se realizó a través de observaciones y entrevistas, con un enfoque interpretativo.

Las evidencias muestran, en este caso, que el liderazgo del director ha sido un factor preponderante, tanto para el éxito de la escuela como para la aplicación del PEC; entre los principales logros que se mencionan están: un trabajo colaborativo de los docentes, mayor participación social, progreso en el logro educativo de los alumnos, y una mejora sustancial en la infraestructura.

Este tipo de estudios es importante para dar cuenta del impacto de una política pública en una escuela concreta, que además se ubica en un contexto social y económico desfavorable, en la que pareciera que el esfuerzo y la dedicación de su director, impulsado por la propuesta del PEC, han sido la clave del éxito.

Palabras clave: Liderazgo; mejora escolar; eficacia escolar; programa Escuelas de Calidad; estudio de caso.

\begin{abstract}
In Mexico, the inequality in the educational opportunity offers stills a big challenge. Among the policies and programs that have been implemented highlights the Quality Schools Program (PEC), which began ten years ago as an institutional reform proposal and changes in educational management. A case of study was performed with a successful school enrolled in this program, which is located in a rural town in the state of Aguascalientes, Mexico. The oncoming is done through observations and interviews, with an interpretive approach.
\end{abstract}

The evidences show, in this case, that principal's leadership has been a major factor for both the success of the school and the PEC; among the major achievements mentioned are: a collaborative work of teachers, greater social participation, progress in the educational achievement of students, and a substantial improvement in infrastructure. 
This type of studies is important to show the impact of a public policy in a specific school, which also is located in an unfavorable social and economic context in which seems that the effort and dedication of its director, driven by the proposed PEC, have been the key to success.

Key words: Leadership; school improvement; school efficiency; Quality Schools Program; case of study.

\section{PRESENTACIÓN}

Actualmente los sistemas educativos de todos los países buscan ser cada vez mejores en todas las dimensiones que organizan su funcionamiento, y de forma especial, en el logro educativo de los estudiantes; para ello, continuamente se analizan y evalúan los múltiples elementos que se asocian a este logro. Asimismo, la valoración de los alumnos es importante en la toma de decisiones, pues se considera que es un indicador preciso de la formación que los niños y jóvenes están recibiendo para su actuación en la vida, en el presente y en el futuro.

En los últimos años, el tema de la gestión escolar ha cobrado una gran importancia en las agendas públicas de la mayoría de los países. Y es que tanto la investigación educativa, como las evaluaciones nacionales e internacionales, permiten prever que una buena gestión escolar es condición fundamental para mejorar los resultados educativos de los alumnos (Miranda, 2010).

La escuela pública mexicana enfrenta grandes retos, dada la diversidad en la composición y distribución de la población, así como la diferencia en las oportunidades que se tienen de recibir un servicio educativo de calidad (Backhoff, y otros, 2007); veamos un ejemplo a través de un indicador.

De manera natural se tiende a asociar la desigualdad de oportunidades educativas con la desigualdad económica; el medio rural de México es en el que se manifiestan los mayores niveles de desigualdad y donde la situación de la pobreza es más aguda. A pesar de haberse logrado algunos avances en los últimos años, un porcentaje muy alto 
de la población de este sector sigue siendo afectado por la pobreza. En 2006, cerca de 55 por ciento de la población rural (poco más de 12 millones de personas) estaban en pobreza de patrimonio. En términos de cobertura educativa, el sector rural está rezagado con respecto del resto del país. A 2005 la escolaridad promedio, medida en años, era de 8,9 para el sector urbano y de 5,6 para el sector rural. Además, en el medio rural no se alcanzan en promedio todavía los años de escolaridad que el sector urbano ha logrado más de una década antes.

Continuando con el ejemplo, los niveles de escolaridad de las personas del medio rural son en general inferiores a los del medio urbano. Según las cifras del II Conteo de población y vivienda 2005, 72 por ciento de la población rural o no tiene instrucción o sólo alcanza la primaria como máximo nivel de escolaridad. En contraste, 55 por ciento de la población urbana tiene un nivel de instrucción de, al menos, la secundaria (Ordaz, 2009).

Mientras que prácticamente el 100 por ciento de los niños que viven en las ciudades y en zonas urbanas tienen acceso a la educación básica —que incluye 12 años de escolarización (preescolar, primaria y secundaria) - en las comunidades rurales y en las indígenas este indicador puede estar muy por debajo, y está altamente diferenciado considerando zonas geográficas y composición poblacional.

Condiciones como la descrita antes, llevan a la escuela a asumirse como promotora de cambio y de transformación social, lo cual fortalece su misión de cara a las nuevas demandas de la sociedad a la que se debe. También cabe mencionar que éste es sólo un ejemplo de una de las múltiples aristas que presenta el sistema educativo nacional, y que ilustra en parte el reto al que se enfrentan las escuelas y los actores educativos.

Una estrategia que puso en marcha el gobierno mexicano, fue la de impulsar el trabajo de las escuelas que realizaban bien su función, y de apoyar cambios favorables en aquellas que tenían la intención de mejorar, de tal manera que contaran con todo lo necesario para seguir haciéndolo. Así fue como surgió, hace poco más de diez años, el Programa Escuelas de Calidad (PEC), como una propuesta de reforma 
de la gestión institucional y escolar, especialmente para superar los obstáculos que impiden el logro educativo. En él se han involucrado autoridades educativas de todos los niveles de gobierno (federal, estatal y municipal) y las comunidades escolares, en especial a través de estrategias de financiamiento, transparencia en el uso de los recursos y rendición de cuentas a la sociedad.

Sus acciones han estado orientadas a crear las condiciones necesarias para impartir una educación pública tendiente a la equidad, no sólo en la cobertura, sino dando énfasis a la calidad del servicio educativo; atender los rezagos en la construcción, mantenimiento y equipamiento de los espacios escolares públicos; y coadyuvar a fortalecer acciones orientadas a transformar la gestión escolar para que todos los educandos logren aprendizajes significativos para su vida presente y futura.

Antes se mencionó el patrón existente en la mayoría de las comunidades rurales, que incluye pobreza y falta de oportunidades de estudio, para dar mayor valor al caso que se presenta posteriormente, pues el estudio se realizó en una escuela rural en condiciones socioeconómicas desfavorables, y ha sido a través de diversas acciones de gestión educativa dentro del PEC — en especial por un liderazgo eficaz de parte de su director- que se ha posicionado como una escuela de éxito. Veamos a continuación la propuesta de este Programa y posteriormente cómo fue aplicado en esta escuela concreta de un municipio en Aguascalientes.

\section{PROGRAMA ESCUELAS DE CALIDAD (PEC)}

En México, el tema de la gestión escolar ha tenido un impulso muy importante desde el Acuerdo Nacional para la Modernización de la Educación Básica de 1992, y que sigue vigente en la actual Reforma Educativa, que reconoce a la escuela como el centro del sistema educativo. Posteriormente se vislumbró la importancia de la planificación en la escuela, y como antecedente directo surgió un proyecto de cooperación mixto México-España denominado «La gestión en la escuela primaria», cuya herramienta de planeación fue el Proyecto Escolar orientado a resolver problemas. Su premisa: la intervención 
pedagógica a partir del reconocimiento de un problema principal ubicado en el ámbito del aula y la enseñanza.

Una vez diseñado y puesto en marcha, el principal objetivo del PEC ha sido transformar la gestión de la escuela para mejorar la calidad de la educación básica; se trata de un proceso de cambio a largo plazo, que tiene como núcleo el conjunto de prácticas de los actores escolares - directivos, docentes, alumnos, padres de familia, supervisores, asesores y personal de apoyo-, y busca crear y consolidar distintas formas de hacer que permitan mejorar la eficacia, eficiencia, equidad, pertinencia y relevancia de la acción educativa (SEP, 2010).

El PEC define a una escuela de calidad como aquella que asume en colectivo la responsabilidad por los resultados de aprendizaje de todos sus alumnos; se compromete con la mejora continua de los procesos y de los resultados educativos; se constituye en un centro seguro y útil a su comunidad que cuenta con infraestructura, equipamiento y tecnología de vanguardia; contribuye con equidad al desarrollo integral de sus alumnos para que desarrollen las competencias requeridas para alcanzar una vida plena que les permita una convivencia democrática, participación en el trabajo productivo y aprender a lo largo de su vida (SEP, 2010).

La innovación que incorporó el PEC en la política educativa consistió, precisamente, en centrar su atención en la escuela y las comunidades que ésta forma para impulsar, desde sus actividades cotidianas, un modelo de autogestión que contribuya a fortalecer el proceso de enseñanza-aprendizaje, considerando que es la comunidad educativa quien decide sus prioridades y puede orientar el presupuesto del que dispone para alcanzar tales objetivos, es decir, para atender lo que realmente necesita.

Es necesario ubicar puntualmente en un contexto histórico y social el surgimiento de este programa; para ello, se retoman algunas ideas de Cárdenas (2010). Es necesario recordar que el PEC fue diseñado tras el arribo de un Gobierno emanado de un partido político diferente al que había gobernado nuestro país por décadas. Este cambio representó una oportunidad para explorar, en el sistema escolar, herramientas que 
habían funcionado en otros campos y podrían abrir oportunidades para incrementar la efectividad escolar.

Asimismo, las condiciones del sistema educativo obligaban a buscar intervenciones ambiciosas, con escasas referencias previas y que en principio acercarían nuevas soluciones a viejos problemas. Por ello, y en concordancia con lo intentado en muchos otros países, se buscó explorar los efectos que tendría implementar un modelo de administración centrada en la escuela, que para el PEC se expresaba como una «reorientación» de la gestión estatal y federal hacia las escuelas, la provisión de recursos para el ejercicio directo en éstas, procurando una mayor participación social y generando una cultura de planeación y evaluación en las mismas.

Los fundamentos del Programa son los siguientes:

Desde 2003, el PEC impulsa un modelo sustentado en los fundamentos de la gestión educativa estratégica y que se explica mediante tres conceptos claves: reflexión, decisión y liderazgo. Su finalidad es integrar una nueva forma de entender y conducir la organización escolar. También se apropia de referencias del movimiento de reforma educativa denominado "gestión centrada en la escuela» (Murnane, 2006), modelo popular en todo el mundo desde los años 90, y que parte de algunos supuestos como los siguientes: facultar a las comunidades escolares en la toma de decisiones, redundará en el planeamiento de la mejora escolar; la asignación de facultades favorecerá la rendición de cuentas; y el empoderamiento de las escuelas generará un modelo de planeación participativa en el que los maestros, directores y padres de familia asumirán mayores responsabilidades respecto de las actividades educativas en beneficio del aprendizaje de los alumnos (SEP, 2010).

Para lograr su propósito, el PEC ha generado un Modelo de Gestión Educativa Estratégica (MGEE), que organiza y da sentido a todos los componentes de la gestión educativa que se ponen en juego de manera intencionada para la mejora escolar. De acuerdo a este modelo, la gestión en el campo educativo se ha clasificado, para su estudio, en tres categorías de acuerdo con el ámbito de su quehacer y con los niveles de concreción en el sistema: institucional, escolar 
y pedagógica (SEP, 2010). Las principales características de la gestión educativa estratégica, que involucra las categorías anteriores, son:

a) Centralidad en lo pedagógico.

b) Reconfiguración, nuevas competencias y profesionalización.

c) Trabajo en equipo.

d) Apertura al aprendizaje y a la innovación.

e) Asesoramiento y orientación para la profesionalización.

f) Culturas organizacionales cohesionadas por una visión de futuro.

g) Intervención sistémica y estratégica.

El MGEE se conformó en el contexto del sistema educativo nacional, con base en las tendencias y las recomendaciones del ámbito local e internacional, con el propósito de apoyar a los centros escolares en la mejora de la calidad de los servicios que ofrecen y los aprendizajes de los estudiantes, a partir del desarrollo de las competencias de todos los actores escolares para la práctica de liderazgo, trabajo colaborativo, participación social responsable, planeación estratégica, evaluación para la mejora continua, como asuntos claves de gestión para enfrentar los retos globales del siglo XXI, en un marco de corresponsabilidad, transparencia y rendición de cuentas (SEP, 2010).

Para la definición de sus particularidades, el MGEE se integra por diversos elementos que le dan sentido y soporte; entre ellos se encuentran sus componentes, los cuales se vinculan e interrelacionan, y son referentes fundamentales para comprender los hallazgos del presente estudio:

1. Liderazgo compartido.

2. Trabajo colaborativo.

3. Planeación estratégica.

4. Participación social responsable.

5. Evaluación para la mejora continua.

El ejercicio de los componentes del PEC y su MGEE ofrece amplias oportunidades para dialogar, compartir observaciones y preocupaciones, y construir o fortalecer el trabajo colectivo entre actores educativos. Es también útil para modificar las formas de ver y entender lo 
que acontece en el hacer cotidiano (SEP, 2010). Cabe mencionar que, actualmente, se está generalizando la aplicación de este modelo a la totalidad de las escuelas de educación básica, y no sólo a las escuelas que pertenecen al Programa, por lo que ya podemos considerarla como una política de influencia nacional, y no sólo como un programa concreto que atiende necesidades específicas. Ante esto, es importante dar cuenta de lo que ocurre en algunas escuelas que lo han aplicado y han tenido buenos resultados, para ilustrar los logros que son posibles.

\section{MÉTODO}

El propósito de esta investigación fue dar cuenta de una experiencia exitosa en la aplicación del PEC que, como ya señalamos, es un programa del gobierno federal que busca la mejora escolar en varias dimensiones de un modelo que propone para tal fin; para ello, se eligió una escuela en el estado de Aguascalientes (centro-occidente de México), que participa en el Programa desde sus inicios (ciclo escolar 2001-2002) y que muestra buenos resultados con su aplicación.

Se realizó un estudio de caso, que es un método de investigación cualitativa que busca comprender en profundidad la realidad social y educativa, en el que se hace una descripción y análisis detallados de unidades sociales o entidades educativas únicas. Es un estudio intensivo de un caso o una situación con cierta intensidad, visto como un «sistema acotado», por los límites que precisa el objeto de estudio, pero enmarcado en el contexto global donde se produce (Stake, 2005).

Para el levantamiento de los datos se realizaron varias actividades: cinco observaciones en la escuela; tres entrevistas con maestros, dos entrevistas con el director y dos con madres de familia, y se analizaron los resultados del centro escolar en diferentes evaluaciones nacionales y estatales. Llama la atención que la institución se localiza en un ambiente social y económico desfavorable, en un contexto rural con situaciones familiares desintegradas, especialmente afectadas por la migración de los jóvenes y adultos (varones) hacia los Estados Unidos.

El marco desde el cual se analizaron las evidencias fue el de los componentes del MGEE, que es el insumo básico generado por el PEC para generar el cambio escolar. A partir de ellos, se realizó un análisis de 
contenido de las entrevistas y los registros, identificando además de la presencia y frecuencia de las categorías analíticas originales, algunas categorías emergentes, así como la intensidad o el énfasis que se ponía en algunas ideas, lo que brindó la pauta para la identificación de los elementos fundamentales para la mejora de la comunidad educativa y el éxito de la escuela.

\section{RESULTADOS}

Acerca del PEC, los hallazgos de las dos evaluaciones de impacto realizadas en el año 2006 por encargo del gobierno federal, y de los múltiples estudios relacionados con el Programa, sugieren que ha logrado efectos positivos en indicadores básicos: en diversos casos ha modificado la cultura escolar, generado nuevas dinámicas de cooperación entre municipios, entidades federativas y el gobierno central, además de provocar debates sobre la rendición de cuentas en nuestro sistema (Cárdenas, 2010).

Desde la perspectiva del PEC, las escuelas son organizaciones que aprenden y son capaces de revisar los alcances de sus prácticas de gestión; por ello, ha buscado establecer criterios y procedimientos que sirven de guías para orientar las acciones que emprende el colectivo escolar. La propuesta del PEC considera que si los maestros, directivos, alumnos y padres de familia forman una auténtica comunidad escolar, ésta tendrá la capacidad de identificar sus necesidades, problemas y metas realizables orientadas hacia la mejora de calidad del servicio educativo (Miranda, 2010).

A partir de las observaciones y de las entrevistas realizadas, se presentan algunos de los resultados obtenidos en este estudio de caso.

Cuando se entra en la escuela Abraham Cruz, la sensación es que se ha llegado a un lugar de trabajo, a un lugar con historia, y al mismo tiempo, a un espacio donde ocurren cosas nuevas. Primero, a uno de trabajo, porque por donde quiera se observa gente que hace algo y que en toda su expresión demuestra que sabe lo que debe realizar. Segundo, con historia, pues el estilo del edificio y la estructura general de la escuela hace pensar en décadas de tradición y en cientos de niños que 
han pasado por sus aulas y espacios. Tercero, en el que ocurren cosas nuevas, pues en buena parte de las instalaciones y en la forma de actuar de las personas, es notable observar que algo importante pasa ahí.

La escuela Abraham Cruz se ubica en la comunidad de Tepezalá, que es la cabecera del municipio del mismo nombre. Se localiza al norte del estado de Aguascalientes, a 47 kilómetros del municipio capital, en los límites con Zacatecas, y es uno de los espacios sociales con mayor rezago y pobreza, lo que ha llevado a buena parte de su población a migrar, tanto a otros municipios y ciudades de la región, como otros lugares fuera del estado, incluyendo los Estados Unidos.

La elección de este centro educativo como experiencia exitosa se debió a dos motivos: 1) su permanencia constante en el PEC, pues es una de las pocas escuelas en el estado que se ha mantenido en el programa desde 2002 (se habla de sólo tres escuelas, y las otras dos están en la ciudad, en ambientes con buenas condiciones generales), y 2) que a pesar de su situación marginal ha tenido buenos resultados, pues se ubica en un ambiente social y económico desfavorable, en un contexto rural con muy bajos ingresos económicos, con pocas posibilidades laborales y con situaciones familiares un tanto desintegradas, todo ello afectado especialmente por la migración ya mencionada.

La escuela cuenta con nueve grupos de $1^{\circ}$ a $6^{\circ}$ grados, con un promedio de 27 alumnos por grado y un docente por grupo, así como dos profesores de inglés, uno de educación física, personal administrativo (1) y de apoyo (5). Una de las principales fuentes en este estudio fue el profesor Tomás Díaz, que funge como director de la escuela desde el año 2000.

Durante una de las visitas realizadas al centro educativo, la idea central expresada tanto por el «profe» Tomás (así es conocido dentro y fuera de la escuela), como por los maestros entrevistados, es que mucho del trabajo en la dirección y de los propios maestros en sus aulas, con los padres y en la escuela, durante los casi doce años que el profe Tomás lleva en esa función y los diez de participar en el PEC, ha sido el de gestionar la existencia de las condiciones necesarias para que el aprendizaje de los niños sea lo central, lo cual es coincidente 
con lo señalado por el PEC, en cuanto a que las escuelas que participan asumen en colectivo el compromiso de mejorar los resultados de aprendizaje de todos sus alumnos (SEP, 2010).

Para ello, hay coincidencia en los testimonios de los actores entrevistados (director, maestros y padres de familia) de que el trabajo se ha basado en un liderazgo efectivo de parte del director y en la generación de un ambiente de confianza en el que todos han participado y han puesto su «grano de arena», logrando mejoras sustanciales en la infraestructura, la existencia y el ejercicio de procesos de participación social (pues antes era prácticamente nula), y un fuerte compromiso de su equipo docente para enseñar, logrando con ello, ya desde hace algún tiempo, la meta que se planteó al inicio de su gestión: que la escuela Abraham Cruz sea reconocida como una escuela de calidad.

\section{Un poco de historia.-}

La escuela fue construida en 1951 por un gobernador del estado de Aguascalientes que era nativo de Tepezalá; dice la historia que en un acto deportivo anunció esta obra "para su pueblo, pues quería llevar la educación a su gente». Así, se edificó la escuela, siendo una de las mejores de su tiempo en cuanto a equipamiento, pues incluso contaba con una planta de luz, así como por el tamaño y la calidad de la construcción, pues en el estado sólo existían tres con la infraestructura que ésta presentaba (las otras dos estaban en el municipio de la capital). Sin embargo, un dato que llama la atención es que pese a la fuerte inversión realizada, la primera generación que egresó fue de sólo tres alumnos.

A partir de ese momento, y dadas las condiciones desfavorables no sólo del municipio sino de la región, la escuela fue cayendo en un estatus poco alentador, con muy poco mantenimiento, y en años recientes, con muy baja demanda de parte de los padres de familia para inscribir a sus hijos en ella. Así, para septiembre del año 2000 que comenzó su gestión el profe Tomás, la escuela estaba en pésimas condiciones físicas: las butacas eran de la época de fundación de la escuela (tenían 50 años), no había energía eléctrica, los pizarrones ya no servían («nomás patinaba el gis») y faltaban cerca de 300 vidrios. 
En lo académico, había índices altos de reprobación y deserción, y bajos resultados en el logro académico, con un colectivo docente desintegrado y participación social nula (reuniones generales sólo con 10 o 15 padres de familia). La población estudiantil era de 196 alumnos en 6 grupos.

Cabe mencionar que el profe Tomás estuvo como docente en esta misma escuela de 1995 al año 2000; durante ese tiempo, se dio cuenta que las cosas podían, y debían, ser diferentes. En la entrevista expresa así su pensamiento de ese momento: «Si tuviera la oportunidad de ser director todo esto cambiaría».

\section{Transformación a partir del PEC.-}

El PEC surgió en el ciclo escolar 2001-2002, y una de sus características era la posibilidad de lograr una inversión hasta por \$300,000 pesos; ante ello, el profe Tomás y su colectivo docente vieron una gran oportunidad de sacar adelante la escuela; durante las entrevistas, y recordando ese momento, tanto el director como uno de los docentes que permanecen hasta la fecha en la institución, mencionan que pensaron que la escuela no sería seleccionada durante el proceso por las pésimas condiciones en que se encontraba.

Con gran ánimo, en la escuela comenzaron a trabajar con proyectos en cada uno de los ámbitos, que era lo señalado por el PEC en ese momento, y la dinámica institucional comenzó a cambiar, dándose un proceso de transformación gradual. Al inicio, los docentes mostraron indecisión y se debió realizar un fuerte trabajo de convencimiento - entre ellos y de parte del director-, basado en un liderazgo efectivo y una adecuada gestión, orientados a los objetivos propuestos.

En este sentido, el trabajo del profe Tomás se centró en lograr un proyecto conjunto, un sentido de cohesión entre todo el personal, partiendo del propio ejemplo en cuanto al compromiso con el trabajo en la escuela; en la segunda entrevista nos comparte una muestra de esto: afirma que cuando llovía y se hacían charcos en la escuela, el director era el primero en ponerse a barrer para eliminar el agua en el patio y la explanada, y al verlo, tanto el personal de limpieza como los mismos docentes y alumnos comenzaban a barrer para dejar lo más seco posible. 
Al preguntarle a los docentes y a las madres de familia qué hace el profe Tomás, coinciden en que quizá mucho de su compromiso se deba a su permanencia en la escuela, pues «casi siempre está ahí y nos atiende» (señala una madre de familia); dicen que es el primero en llegar, trata de estar lo más posible en la institución durante su jornada laboral, a pesar de las múltiples actividades de gestión que realiza fuera de la escuela; y mientras se encuentra ahí, procura atender de forma lo más eficiente posible tanto las contingencias que surgen en los grupos, como con los padres de familia que continuamente asisten, y da seguimiento a las actividades cotidianas de todo el personal.

Con acciones y actitudes como éstas — que conforme a la perspectiva del PEC son propias de un liderazgo comprometido, el cual se refleja en un conjunto de prácticas de los actores escolares (SEP, 2010) - y con un colectivo bien dirigido a metas concretas y pertinentes, tanto para un ambiente adecuado de trabajo como para el aprendizaje, congruentes con la realidad del centro escolar, el director logra mover a la organización, pues «invita» a los demás a ponerse la camiseta, a comprometerse, y con ello, la comunidad escolar tiene un sentido de claridad en el rumbo trazado: el esfuerzo y la constancia de todos lleva al éxito en las tareas cotidianas y al cumplimiento del deber ser de la escuela.

En el momento actual, y gracias al PEC (mediante los procesos de cambio que ha generado a través de la exigencia en el cumplimiento de indicadores), la institución cuenta con una población cercana a los 300 alumnos distribuidos en 9 grupos, llegando con ello al máximo de su capacidad. Una maestra comenta: «Ya no podemos aceptar a más niños, aunque los papás se enojen, porque ya no tenemos sillas dónde sentarlos... y además, luego las otras escuelas se van a quedar vacías y qué van a hacer».

Otro elemento que cuida el PEC es la infraestructura, pues plantea que una escuela de calidad es aquella que cuenta con equipamiento y tecnología de vanguardia; en este sentido, un logro importante que los docentes y el director le atribuyen al PEC es la sala de cómputo, la cual se abasteció con equipo reciente y se le dio mantenimiento y adecuación, de tal manera que ahora sí se le da un uso constante. 
Asimismo, y como parte de los resultados obtenidos gracias a Escuelas de Calidad - pues los docentes reconocen en las entrevistas que por su inclusión en el Programa se aplican con mayor compromiso a lograrlo-, se han reducido a un mínimo los indicadores de deserción y reprobación, y se ha elevado el nivel de logro académico de los alumnos (por ejemplo, con mejores resultados en la prueba ENLACE y en las Olimpiadas del Conocimiento). Así, el aprendizaje de los alumnos, a partir del esfuerzo del profe Tomás y el convencimiento que logró en el colectivo docente, pasó a ser una prioridad en los planes de trabajo y en las actividades realizadas.

Un aspecto del MGEE, que propone el PEC, es que el ejercicio de los componentes de este modelo ofrece oportunidades para dialogar y fortalecer el trabajo colectivo entre los actores educativos; así, tenemos que otro ejemplo de gestión y liderazgo pedagógico es la promoción de una formación continua y continua capacitación del personal docente; para ello, el director ha facilitado que los profesores asistan a cursos y diplomados, y señala que «la única condición que les pongo, y siempre los comprometemos [a los maestros], es de que cuando regresen, deben compartir con todos y aplicar lo que fueron a aprender».

En el caso de los docentes, a medida que se han ido convenciendo del cambio de actitud y se ha vencido la resistencia al cambio, en un proceso «incluso doloroso» (entrevista con una docente), las reuniones de Consejo Técnico Escolar ahora son verdaderamente de carácter técnico-pedagógico y no de tipo social, con temas propuestos por ellos mismos, que les favorecen en su profesionalismo y, de forma central, a los alumnos en su logro académico. De acuerdo a las palabras de uno de los docentes: «Los maestros se han convertido en la parte viva de este proceso y están totalmente convencidos».

En este sentido, recientemente algunos profesores generaron una experiencia innovadora para la identificación de los estilos de aprendizaje en los alumnos y el diseño de una evaluación didáctica pertinente, que luego se generalizó a toda la escuela; cabe mencionar que de acuerdo con el informe presentado acerca de esta experiencia, desde hace siete años los docentes dejaron de adquirir pruebas comerciales con las cuales evaluaban a sus alumnos, y ante ello, se propusieron - como 
colectivo docente- generar instrumentos de evaluación congruentes y pertinentes a la realidad educativa de sus estudiantes, buscando que también se reflejara en una planeación didáctica eficaz, para alcanzar mejores resultados.

Así, durante los últimos ciclos escolares, y de acuerdo a los análisis que se fueron realizando en las reuniones de Consejo Técnico, de los diversos tipos de instrumentos que se fueron aplicando y de los resultados de logro académico (que eran muy bajos al compararlos con los resultados de las evaluaciones externas), se dieron a la tarea de re-estructurar la estrategia de elaboración de instrumentos de evaluación. Debido a ello, se estableció una dinámica diferente donde se llegó al acuerdo de elaborar evaluaciones que se asemejaran a la evaluación externa.

El resultado de esta decisión fue un enfoque cooperativo de la evaluación en un clima pleno de confianza, en el cual se promovió entre los docentes la creación de estrategias internas de evaluación, adecuadas a la realidad de los alumnos (sus estilos de aprendizaje), mismas que permitieran la creación de instrumentos pertinentes y eficaces, y provocaran una evaluación interna más productiva (relacionada directamente con las planeaciones didácticas de los docentes), a partir de los intereses de la institución: elevar el nivel de logro académico en las evaluaciones externas. Y los resultados no se hicieron esperar.

Una evidencia contundente es la siguiente: el puntaje obtenido en la prueba ENLACE para $5^{\circ}$ grado, en la materia de Español, pasó de un promedio de 535 en 2007 a 695 en 2010, y en el caso de Matemáticas, el salto fue de 517 en 2007 para llegar a 630 en 2010. De igual manera, si consideramos los resultados en ENLACE de $3^{\circ}$ a $6^{\circ}$ grado en cuanto al puntaje ganado en Español y Matemáticas, entre los ciclos 2006 y 2010, va de 122 a 271 ; además, hay que considerar que en todos los grados hubo ganancias (es decir, en ninguno existió una pérdida o retroceso en el puntaje promedio).

A medida que fueron cambiando las cosas - y por la presión positiva que ejercía el PEC - , fue mayor el compromiso de todos los involucrados, resultando en dos efectos positivos: un cambio favorable en todas las dimensiones de la realidad educativa y un beneficioso impacto social. 


\section{Hacia una participación activa de la comunidad.-}

En torno a la participación de los padres de familia y la comunidad, de acuerdo a lo mencionado tanto por los padres de familia como por el director, el Consejo Escolar ya es una realidad social activa y no sólo de membresía (pasiva), generando una escuela incluyente, con una amplia gama de valores que aportan todos los actores involucrados (docentes, alumnos, padres de familia, ex alumnos, líderes de la comunidad), lo cual, desde las perspectivas del director y el equipo docente, fortalece todos los procesos.

Ahora, lo que pasa en Tepezalá es que todos quieren tener a sus hijos en esta escuela, a pesar de que existen otras dos instituciones públicas en la comunidad. Las reuniones con padres de familia son en la sala de usos múltiples y presentan una participación de 150 a 160 personas cada vez. Una madre de familia, durante la visita a la escuela, se expresó de la siguiente manera:

"Yo no soy de aquí, pero desde que llegué, los comentarios que escucho sobre esta escuela es que es la mejor; incluso, cuando los niños van a ingresar por primera vez, es muy peleado el lugar, es mucho muy peleado. Uno como mamá tiene que andar atrás del maestro [Tomás] para ver cómo va a estar [el proceso] para que nos toque lugar, porque es mucho muy peleado para estar en esta escuela».

El profe Tomás comenta que cuando él era profesor y de recién llegado a la dirección, la participación de las madres en las reuniones era muy pobre, con sólo 10 o 15 personas, y entre ellas había muchas discusiones y pocos acuerdos. Se puso la meta, junto con los docentes, de que asistiera alguien de la familia por cada alumno de la escuela, y parece que lo han logrado; incluso señalan que ahora las reuniones deben ser en la explanada porque la sala de usos múltiples resulta insuficiente.

El proceso que se llevó a cabo fue en dos sentidos: desde la perspectiva de un liderazgo compartido y una invitación personalizada de parte del director; respecto del liderazgo, la lógica de las reuniones cambió de ser vertical y excluyente, a ser horizontal e inclusiva; de ser meramente informativa y sólo con algunos representantes de cada 
grupo, a una en la cual todos pudieran dar su opinión y en la que se privilegiara la diversidad, el análisis de las propuestas y la elección de las mejores opciones, todo con base en el diálogo y el respeto. El profe Tomás reconoce que esto no es fácil, y que no siempre da gusto a todos, pero que es la única forma de que participen y se genere un sentimiento de «vamos a hacerlo por nuestra escuela».

Una estrategia que empleó el director, y que trascendió a los docentes, fue hacer invitaciones verbales personalizadas, en las cuales, el conocimiento de la comunidad y de quienes viven en ella fue fundamental. Así, los padres de familia comentan que no sólo les enviaban el citatorio para las reuniones, sino que iban a platicar con ellos a su casa (los docentes o el director), y de no encontrarlos, les hacían llegar con conocidos y vecinos la noticia de que sin ellos no estarían completos, de que ahora las cosas eran de «todos o ninguno». Esto, aunado a la toma de opiniones y el consenso, generó una sinergia positiva y una actitud favorable hacia la escuela y lo que en ella se hace, de tal manera que ahora todos participan, y quienes no pueden hacerlo por cuestiones laborales $\mathrm{u}$ otros motivos, de todos modos buscan estar informados y participar.

Otro de los elementos importantes de la participación social en esta escuela, que se vincula con el liderazgo del director, radica en el compromiso de algunos líderes de la comunidad, entre ellos de varios políticos y servidores públicos pertenecientes al ayuntamiento en turno. El profe Tomás comenta que, independientemente de la adscripción partidista de quien se encuentra dirigiendo el municipio, una habilidad que tiene es la de ser buen negociador, y por ello, ha podido mediar entre diferentes personas quienes tienen influencia tanto en la comunidad como en la administración de los recursos, y con ello ha logrado apoyos importantes para la escuela.

Como ejemplo menciona la primera participación en el PEC, para la cual era necesario reunir una cantidad de dinero, la cual se fue sumando con diferentes aportaciones del presidente municipal, un diputado, y el presidente del consejo estatal de participación social; y una vez lograda esa parte, con las aportaciones de dinero de los padres de familia, a quienes se les indicó que, si otras personas habían 
hecho aportaciones a la escuela, era importante que ellos que tenían a sus hijos ahí, participaran con lo que pudieran para alcanzar la meta. Y el objetivo se alcanzó, pues se reunió la cantidad requerida y la escuela pudo participar en esa convocatoria del PEC.

Ahora se dice que nadie le puede negar nada al profe Tomás porque sabe cómo pedirlo, porque presenta evidencias contundentes del trabajo realizado y porque sabe comprometer a las personas con lo que la escuela requiere. Además, una vez conseguida la meta propuesta, hace partícipes a todos del éxito alcanzado y les invita a ver lo que se ha logrado, tanto en actos cívicos como de manera personal, de tal forma que ahora, en la escuela, tanto alumnos como docentes están acostumbrados a la presencia de personas que van a observarlos para saber qué están haciendo.

Un ejemplo de mejora en la infraestructura: el desayunador.-

Uno de los aspectos más visibles en esta escuela es el buen estado general de sus instalaciones; a pesar de contar con una estructura algo anticuada (con 60 años desde su construcción), tiene un buen mantenimiento y está muy limpia.

A partir de la participación de la escuela en el PEC, y aprovechando los recursos de que pudieron disponer, la infraestructura mejoró considerablemente. Con el primer apoyo se pintó toda la escuela, se cambiaron todos los vidrios, se arreglaron todos los pizarrones y se colocaron pintarrones; además, se construyó la sala de usos múltiples.

Ahora todos los salones tienen sillas y mesas buenas; se crearon áreas verdes donde antes había "pura tierra»; se colocó un asta bandera en el patio cívico; se dispusieron gradas y sombras, y la totalidad de las instalaciones está bien pintada. En palabras del director y de los docentes, ahora «las condiciones son óptimas para el desarrollo de nuestros eventos».

Cuando los padres de familia y la comunidad escolar advirtieron que lo que pretendían el director y los maestros se hacía realidad, empezó a generarse un impacto de compromiso compartido. Afirmaban 
en las entrevistas: «si ellos [PEC, director, docentes] están dando, no es posible que nosotros nos quedemos en nada y continuemos haciendo lo mismo». A partir de ahí, surgió el cambio.

El profe Tomás sintetiza este principio de la siguiente manera: «Necesitamos generar un ambiente agradable, que se pueda establecer un lugar donde se pueda aprender de manera armónica, para poder sentirnos a gusto. Así, si generamos condiciones óptimas para un buen desempeño y el niño viene con ganas de venir a la escuela, el maestro viene con esa actitud positiva de venir y trabajar, las cosas cambian y pronto vamos a ver [mejoras] en el rendimiento académico y lo vamos a reflejar en los resultados».

En este proceso, reconocen todos (director, docentes y padres entrevistados) que la optimización de los recursos y la rendición de cuentas son indispensables, porque un ejercicio digno y el trabajo mismo generan un ambiente de confianza, y por tanto, un compromiso compartido. En México, el ambiente de corrupción presente en todos los niveles, no permite el crecimiento de las diversas comunidades, incluso las educativas; por ello, estos actores educativos reconocen que la transparencia en el empleo de los recursos, fue una de las bases para que el trabajo se fuera realizando en un ambiente de confianza y optimismo.

Un ejemplo concreto acerca de la necesidad de contar con las condiciones materiales necesarias, es la habilitación de un espacio en la escuela a manera de desayunador, en el que las madres de familia pueden llevar el almuerzo a sus hijos de una manera adecuada.

En buena parte de las escuelas del Estado — sean de turno matutino o vespertino-, durante el recreo, los niños toman algún alimento que les es llevado por las mamás y que les entregan a través de las mallas perimetrales de los centros escolares o por la puerta de acceso. En este caso, la idea del desayunador consistía en un espacio en donde se colocaron mesas y bancas de cemento en una zona de la institución que cuenta con muchos árboles y con acceso libre para las madres de familia, durante el recreo. Este acceso es vigilado por uno de los profesores, quien hace guardia durante el recreo. 
Durante las observaciones pudimos ver que, en cuanto al funcionamiento, niños y mamás saben que ese espacio tiene una finalidad y hacen uso respetuoso del mismo, no traspasando sus límites o dejando basura en él. Esta acción ha generado un espacio adecuado donde es posible la interacción entre madres e hijos para consumir alimentos a mitad de la jornada respetando, en este sentido, las costumbres de la comunidad.

Los resultados indican la gran importancia del liderazgo ejercido en la institución, a través de su director, el profesor Tomás. A partir de lo que el PEC promueve y genera, apuntaló una forma de trabajo colaborativo entre el personal, una mayor participación social en las actividades de la escuela (especialmente con las madres de familia), una mejora sustancial en la infraestructura y un avance esencial en el logro de los estudiantes.

\section{CONCLUSIONES}

Durante el trabajo de campo realizado, mientras se elaboraba el análisis de la información recolectada y conforme a los hallazgos presentados, se pudo reconocer que una figura clave en el funcionamiento de esta escuela es el director. Las evidencias muestran que el ejercicio de la función directiva es de carácter integral y representa una tarea compleja que requiere del apoyo de la comunidad escolar, para lograr tanto las metas institucionales como el éxito académico de los estudiantes. No se limita únicamente al cumplimiento de las tareas administrativas, sino que se manifiesta en varios niveles de concreción en el sistema: institucional, escolar y pedagógica (SEP, 2010), pues también se enfoca en solventar las necesidades pedagógicas de los alumnos, promover la formación continua de los docentes, hacer partícipe a la comunidad escolar de las decisiones, y dar seguimiento a las actividades educativas de los alumnos y profesores, entre otras acciones (Aguilera, 2011).

Al cuestionar al profe Tomás qué ha aportado el PEC a su escuela, responde que lo ha hecho de dos maneras: en lo económico, ha redituado directamente en la infraestructura y las condiciones materiales necesarias, y esto ha sido la base de la transformación escolar; y ofreciendo una forma procesual de ver y hacer las cosas, en la cual 
todos los actores están involucrados, en donde todos están relacionados mediante un trabajo colaborativo y son co-responsables de lo que pasa, buenos o malos resultados, y en donde todos saben lo que hacen los demás. Así, ha impactado la parte formativa del proceso escolar (de docentes y alumnos) y ha marcado un rumbo claro y bien definido.

Por lo que se puede observar en el centro educativo, un factor indispensable y de gran peso, es el compromiso generado por el director y el equipo docente, quienes en su mayoría viven en la misma comunidad y se han convertido en «buenos profetas en su propia tierra»; incluso el profe Tomás ha pasado buena parte de su vida en esta escuela, pues ha vivido 24 años en la misma (seis años como estudiante de primaria, dos años como estudiante de secundaria que en ese momento compartía las mismas instalaciones, cinco años como profesor y once años como director).

La forma de trabajo instaurado en la escuela Abraham Cruz es la del "placer y la norma», es decir, en la que sea evidente el gusto por hacer las cosas y tomar conciencia de la responsabilidad que conlleva la labor que cada uno realiza. El profe Tomás describe así el compromiso que se genera con este sistema de trabajo:

«Tú maestro, tú alumno, tú padre de familia, ven... porque eres el mejor maestro, el mejor alumno, el mejor padre de familia y vienes a la mejor escuela y tienes al mejor director... ven con gusto y con responsabilidad a hacer tu trabajo porque vida solamente tenemos una. Y cuando hay una transgresión, se aplica la norma, porque la norma es tajante y se debe aplicar sin miramientos; pero es mejor trabajar por gusto y por placer».

El director de esta escuela considera que si el PEC ha dado resultados en este centro escolar, y si todos «nos ponemos las pilas», al estilo de un liderazgo distribuido (Murillo, 2006), el programa deberá funcionar en todas las escuelas. Y concluye: «Mientras el PEC exista y los padres de familia y nosotros queramos, vamos a seguir avanzando».

Los resultados arrojados por el PEC en sus diez años de aplicación en esta escuela manifiestan la importancia que tiene la mejora de la infraestructura y el equipamiento escolar, y el papel trascendental que 
juegan los colectivos escolares, junto con la participación social, para impulsar la autogestión, actuar de acuerdo con las condiciones internas y las características de su entorno, e impulsar proyectos de transformación escolar que se reflejen en la mejora continua de los resultados educativos de los alumnos (Miranda, 2010).

Considerando que el PEC ha sido parte de una política federal, y parafraseando a Cárdenas (2010) en la evaluación que hace del mismo, consideramos que la mejor manera de aumentar sus resultados no será solamente resaltando sus logros, sino realizando en todos los niveles de gobierno y de toma de decisiones lo que el propio Programa pide a las escuelas: reflexionar, planear e implementar los proyectos de mejora que sean necesarios.

En la mayoría de las políticas estatales para la educación se requieren años para evidenciar su impacto real en el funcionamiento de los centros escolares; en este caso, era importante dar cuenta de una experiencia exitosa de una escuela incorporada al PEC, pues con ella se evidencian las formas concretas en que un programa gubernamental genera cambios reales en las escuelas, aunque se reconoce que esto será así gracias al esfuerzo de sus actores y la visión clara y bien enfocada de un líder con perspectiva de éxito. Los programas y propuestas, tanto oficiales como de los académicos e investigadores, siempre serán aplicados por los actores educativos, quienes tienen cara y nombre concretos. Si logramos que sus motivaciones coincidan con lo que señalan los programas, habremos dado un paso importante en la concreción exitosa de los mismos. 


\section{REFERENCIAS}

AGUILERA, Ma. Antonieta. La Función Directiva en secundarias públicas. Matices de una tarea compleja, Instituto Nacional para la Evaluación de la Educación, México, 2011.

CÁRDENAS, Sergio. «El Programa Escuelas de Calidad: una reflexión a diez de pensar en la escuela como el centro educativo», Educare, año 4, edición especial, 2010, pp. 13-15.

BACKHOFF, Eduardo; BOUZAS, Arturo; HERNÁNDEZ, Eduardo y GARCÍA, Marisela. Aprendizaje y desigualdad social en México, Instituto Nacional para la Evaluación de la Educación, México, 2007.

MACBEATH, John. Future of Teaching Profession. Education International Research Institute, University of Cambridge, 2012.

MIRANDA, Francisco. «El PEC y la agenda pública educativa en México», Educare, año 4, edición especial, 2010, pp. 18-20.

MURILLO, Javier. «Una dirección escolar para el cambio: del liderazgo transformacional al liderazgo distribuido», Revista Electrónica Iberoamericana sobre Calidad, Eficacia y Cambio en Educación, volumen 4, número 4e, 2006, pp. 11-24, en: [http:/ / www.rinace.net/ arts / vol4num4e/ art2.pdf]. Consultado: 1-XII-2012.

ORDAZ, Juan Luis. México: impacto de la educación en la pobreza rural. Sede Subregional de la CEPAL en México, 2009, en: [http:/ / www.eclac.org/publicaciones/xml/4/35044/Serie_105.pdf]. Consultado: 1-XII-2012.

SECRETARÍA DE EDUCACIÓN PÚBLICA (SEP). Experiencias escolares exitosas, Programa Escuelas de Calidad, Subsecretaría de Educación Básica, México, 2008. 
SECRETARÍA DE EDUCACIÓN PÚBLICA. Modelo de Gestión Educativa Estratégica, Dirección General de Desarrollo de la Gestión e Innovación Educativa de la Subsecretaría de Educación Básica, México, 2010.

STAKE, R. Investigación con estudios de caso, $3^{\text {a }}$ edición, Morata, Madrid, 2005.

ZORRILLA, Margarita (coord.). Hacer visibles buenas prácticas. Mientras el debate pedagógico nos alcanza. Consejo Mexicano de Investigación Educativa, México, 2005. 